\title{
Nuevos medios de comunicación y movilización transnacional: el caso del Movimiento Zapatista
}

\author{
Markus S. Schulz*
}

\section{Resumen}

Las nuevas tecnologías de la información y la comunicación facilitaron no sólo una globalización económica acelerada, también fomentaron la esperanza de configurar una robusta sociedad civil y global de cosmopolitas solidarios comprometidos con el activismo transnacional. El levantamiento zapatista en Chiapas ha sido ampliamente considerado como el caso prototipo de conflicto social donde el uso de Internet desempeñó un papel instrumental. Este texto cuestiona e indaga en los supuestos generales sobre el impacto del uso de Internet en dicho conflicto. Para ello, se realizó una investigación empírica de páginas web y listas de correos electrónicos, referida contextualmente a las transformaciones de la infraestructura mediática de México a partir de los años noventa. El artículo hace un llamado a la cautela respecto a la valoración del papel que desempeña Internet como herramienta en los movimientos sociales, matizando aspectos significativos que contribuyen ya sea a sobre o subestimar dicho papel. Para concluir, se presentan varias hipótesis específicas a fin de orientar investigaciones a futuro.

\begin{abstract}
The New Information and Communication Technologies not only facilitated an accelerated economic globalization, they also fostered hopes for a global civil society of solidary cosmopolitans and transnational activism. The Zapatista uprising in Chiapas has been widely considered to be the prototypical case of a conflict in which the Internet had a decisive impact. This paper questions whether Internet's impact was exaggerated and investigates its role empirically through quantitative and qualitative analyses of web sites and electronic mailing lists within the broader context of Mexico's changing media infrastructure since the nineteen-nineties. The findings support a more nuanced account and assessment of the Internet's role vis-à-vis other media interfaces. The paper concludes with a set of general hypotheses for further research.
\end{abstract}

Palabras clave: globalización, Internet, esfera pública, comunicación política, movimientos sociales, activismo transnacional, Zapatistas, Chiapas.

Keywords: globalization, Internet, public sphere, political communication, social movements, transnational activism, Zapatistas, Chiapas.

* Doctor en Sociología por la New School for Social Research. Profesor de la Universidad de Illinois en Urbana-Champaign, Estados Unidos, e investigador asociado al Centro de Estudios sobre América Latina y el Caribe de la misma universidad. 


\section{Introducción}

os nuevos medios de comunicación ${ }^{1}$ juegan un rol paradójico en los procesos de globalización y reconfiguración de lo que comúnmente se conoce como la "sociedad mundial". Dichas tecnologías mediáticas han facilitado, por una parte, la tendencia actual hacia la globalización de los mercados, el comercio y la producción de bienes. Por otra parte, los nuevos medios también son considerados como herramientas que permiten oportunidades de interconexión a una gran gama de grupos sociales ubicados en espacios geográficos distantes entre sí sentando, de ese modo, las bases para la emergencia de una sociedad civil y una verdadera "era global" (Albrow, 1997). La noción de la "aldea global", término que fue acuñado a raíz de dinámicas globales que se dieron en medios de comunicación electrónicos anteriores (McLuhan, 1964), vio una renovada popularidad.

En la actualidad, Internet es considerada como una herramienta prometedora capaz de fomentar la comunicación interactiva transnacional y el empoderamiento de actores e incipientes movimientos locales de base. Mientras que las intranets privadas de empresas transnacionales y el comercio global promueven el crecimiento sin precedentes de transacciones económicas mundiales, se le atribuye a Internet la capacidad de proveer la infraestructura necesaria para la construcción de una cultura cosmopolita capaz de equilibrar el poder desmesurado del mercado, e incluso de remediar la pérdida de capacidad política. Este artículo examinará con detenimiento cómo estas "contra-fuerzas" están utilizando Internet. Para ello, se enfocará en el caso probablemente más publicitado en el que se considera que Internet jugó un rol instrumental: la insurgencia zapatista.

El presente estudio se desarrolla en cuatro pasos. Empieza con un breve análisis del discurso sobre el papel de dichos medios de comunicación en el caso de los zapatistas en México. El cuestionamiento de las ideas aceptadas respecto a estos discursos conlleva, en el siguiente paso, un estudio detenido de datos empíricos. De este modo, un vistazo rápido del ciberespacio mexicano y del proceso de establecimiento de Internet en México, proveerá en la segunda sección del artículo un contexto para investigar la función de aplicaciones específicas: a saber, el papel que juegan los sitios

1 El término nuevos medios se utiliza aquí en referencia a las nuevas tecnologías de información y comunicación, tales como Internet y sus varias aplicaciones. Dichos medios son principalmente digitales, o como mínimo, una mezcla entre lo digital y lo análogo. 
Web en la tercera sección del artículo y el de los correos electrónicos en la cuarta sección. A modo de conclusión, se considerará en formato de discusión una serie de hipótesis a fin de orientar mayor investigación empírica a futuro sobre el potencial y los límites de los nuevos medios de comunicación social.

\section{El discurso de la guerra por Internet}

El levantamiento de campesinos indígenas mayas en el estado mexicano de Chiapas recibió una atención mediática mundial e inspiró un movimiento transnacional a favor de los insurgentes y en protesta contra el Tratado de Libre Comercio de América del Norte (TLCAN) y la globalización neoliberal.

Tanto en el ámbito periodístico como en el académico, existe la noción generalizada de que Internet jugó un papel crucial en la insurgencia zapatista. El activista y estudioso Harry Cleaver (1995), por ejemplo, hizo referencia a un "nuevo tejido electrónico de lucha"; otros activistas - "hacktivistas" o "guerreros de red" como se auto-denominaban- utilizaron repetidamente el término "ciberguerra" para referirse a la dimensión en línea de la lucha. Incluso, un boceto del Subcomandante Marcos representado como "ciberpunk" con pasamontañas, altavoz y computadora portátil circuló en numerosos sitios web.

La prensa internacional se dedicó a diseminar ampliamente la noción de una guerra zapatista por Internet desde las etapas incipientes del movimiento. Robberson (1995), por ejemplo, señaló en el Washington Post que "los rebeldes del Ejército Zapatista de Liberación Nacional [habían] invadido el ciberespacio" y nombró el movimiento de Chiapas "uno de los temas informativos más candentes de Internet". En un artículo anónimo, el periódico The Christian Science Monitor (1995) describió al Subcomandante Marcos como alguien que accedía al mundo en línea a través de una computadora portátil cargada con la pila de un carro, un módem y un teléfono celular, señalando que el poder de los rebeldes se basaba precisamente en el acceso a Internet. Evidentemente, a los autores no se les ocurrió que la guerrilla, por lo menos en esa fase, ni siquiera hubiera querido utilizar la telefonía móvil, aún si hubiera habido tal servicio en las localidades remotas en las que se encontraba, dado que ello habría facilitado su detección por las fuerzas militares del Estado.

El gobierno y los medios mexicanos también adaptaron la noción de guerra por Internet. El periódico Reforma, por ejemplo, tituló en abril de 1995 
un artículo de portada de la siguiente manera: "Chiapas es una guerra de tinta e Internet", citando las palabras del entonces Secretario de Relaciones Exteriores, José Ángel Gurría. Éste había acuñado la frase el día anterior en un encuentro en el World Trade Center en México D.F. con ciento cincuenta dirigentes del mundo de negocios provenientes de treinta y siete países. La intención de Gurría era la de calmar a los inversores extranjeros, reasegurándoles la estabilidad económica y política del país. Su caracterización del conflicto de Chiapas como una guerra de "tinta, palabras escritas e Internet" tenía el objetivo de desestimar la dimensión militar de la lucha armada. El gobierno mexicano mantenía la firme posición de que el levantamiento era algo limitado a tan sólo unos pocos municipios en un estado periférico, que ni una bala había sido disparada después del breve combate inicial y que todo el asunto había sido representado de manera descontextualizada y por ende desproporcionada, por el discurso mediático.

Varios estudiosos reconocidos, cuyos trabajos presentan aproximaciones diversas y hasta opuestas, han profundizado en el asunto sobre la asociación entre los zapatistas y la nueva red. Al desaparecer la Unión Soviética como enemigo principal, las fuerzas militares del imperio estadounidense no sólo vieron sus fuentes sino también la justificación de financiamiento en peligro de zozobrar, por lo cual se dedicaron a buscar nuevas amenazas para justificar gastos en nombre de la seguridad nacional. David Ronfeldt y John Arquilla (1998), investigadores de la Corporación Rand - un thinktank al servicio del Pentágono con base en Santa Mónica-, caracterizaron el conflicto de Chiapas como un nuevo tipo de código de guerra al que llamaron "social netwar", o en español, "guerra de red social". Colocaron a los zapatistas, dada su extensa estructura de red, en la misma categoría que Al-Qaeda y advirtieron sobre su perfil como amenaza emergente mucho antes de los ataques a las Torres Gemelas en Nueva York y al Pentágono en Washington, D.C. Desde una perspectiva más afín a identificar injusticia y opresión, el reconocido sociólogo Manuel Castells se refirió a la insurgencia zapatista en su gran trilogía La era de la información, como la forma prototípica de contención en el surgimiento de la "sociedad red" (2001, 2002). Más adelante, Guiomar Rovira Sancho (2009) criticó la falta de observaciones empíricas en el análisis de Castells e incluso señaló errores de datos y hechos. En su búsqueda de dinámicas sociales de punta, por así decirlo, Castells no prestó la debida atención al contexto histórico del internacionalismo que formaba parte de las redes transfronterizas desde mucho antes (Ishay, 1995; Keck y Sikkink, 1998). Si por un lado, Ronfeldt y Arquilla consideraban el potencial de las nuevas tecnologías como amenaza, Castells, por otro, veía una nueva fuente de esperanza para la lucha 
social. Aún con estos matices, la relación entre zapatistas y la nueva red se establecía y reiteraba como algo dado por sentado, una dinámica natural que no ameritaba mayor exploración. La investigación reciente de Rovira (2009) es una excepción importante que cuida la sobrevaloración tecnológica en las dinámicas del movimiento zapatista, aunque no se enfoque en el papel específico que en él desempeñan las nuevas tecnologías mediáticas.

El levantamiento zapatista también inspiró a artistas de diversos países a experimentar con nuevas formas de expresión en el mundo digital. El Teatro de la Perturbación Electrónica (EDT por sus siglas en inglés), un grupo de artistas de performance digital, organizó una serie de "ocupaciones virtuales" contra el gobierno mexicano y el de Estados Unidos con una herramienta basada en JavaScript llamada "FloodNet" o "inunda-red" zapatista (Domínguez, 2002; Lane, 2003). Si bien estas acciones concentraron públicos más bien pequeños, este tipo de obra artística contribuyó al reconocimiento y la valoración de la dimensión cultural de la lucha.

Años después, luego de la caída del régimen del Partido Revolucionario Institucional (PRI) en las urnas, el entonces nuevo presidente Vicente Fox (2000) elogió a los zapatistas en un discurso en ocasión del lanzamiento de su iniciativa e-México, por sus logros como pioneros en el uso de Internet. Sin embargo, la alusión de Fox fue tomada como una crítica codificada del gobierno anterior, el cual no había logrado sacar adelante ninguna iniciativa de acceso público a las nuevas tecnologías de información y comunicación en el país. La retórica de Fox se basaba en la imagen generalizada de los zapatistas como expertos artífices de la computación y de hecho, sirvió para consolidarla aún más.

La identificación de los zapatistas como peritos de la computación en tanto herramienta clave de lucha social se encuentra tan afianzada en la imaginación colectiva que, incluso, ha sido utilizada por la industria publicitaria comercial. Por ejemplo, un aviso publicitario de página completa para computadoras personales en un periódico alemán de gran tirada, retrataba a un rebelde con pasamontañas contemplando sonriente la pantalla de una computadora. Es evidente que el aviso publicitario buscaba aumentar las ventas del producto basándose en la noción de que dicho tipo de referente iconográfico ya se encontraba generalizado en el imaginario popular al punto que se había descontextualizado y des-localizado lo suficiente como para empezar a establecerse asociaciones más amplias entre experiencia humana y tecnología de punta.

La noción de guerrilleros indígenas armados con el ciberespacio se propagó, saturando tanto la imaginación popular como la de los ámbitos intelectuales. La aceptación de dicha representación se encuentra de for- 
ma generalizada en toda variedad de discursos académicos, lo cual tiende a obstaculizar el cuestionamiento de dicha representación a través de una exploración empírica sistemática. Aunque se acepte que Internet desempeñó un papel significativo en la lucha zapatista, cabría preguntarse cuán decisivo fue éste en realidad. Asimismo, ¿se convirtió el levantamiento zapatista en una "guerra de Internet", es decir con Internet como arma principal, por el mero hecho de catalogarlo así? Hay que recordar que cuando el gobierno mexicano utilizaba la expresión "guerra de Internet", lo hacía para restarle importancia al levantamiento; no obstante, los seguidores zapatistas con pericia informática que se hallaban radicados en países post-industrializados, podían entender dicha referencia como un halago y así sentirse aún más motivados a la luz de esta caracterización de sus actividades.

Irónicamente, los polos opuestos de contextos sociales y culturales dieron lugar a una coincidencia entre enemigos que pudo haber dado lugar a una sobrevaloración del rol e impacto que efectivamente tuvo Internet en el levantamiento zapatista. Es por ello que se ha de realizar una consideración empíricamente más sistemática del papel que jugó Internet en el conflicto, a fin de entender con mayor claridad cómo se le utilizó. En las siguientes secciones de este artículo se estudiarán con mayor detalle los diferentes aspectos de Internet, no para imponer un determinismo tecnológico o supuestos ontológicos, ${ }^{2}$ sino con el objetivo heurístico de comprender mejor este rol. El apartado a continuación considera el establecimiento de los nuevos medios en México a partir de la primera mitad de los años noventa. ${ }^{3}$

\section{El ciberespacio mexicano en contexto}

Internet ha crecido exponencialmente durante las décadas recientes. El número de usuarios a nivel mundial aumentó de 2.2 millones de servidores en enero de 1994 a más de 768 millones hacia julio de 2010, según el conteo de Network Wizards (ISC, 2011). Desde 1993, el desarrollo de interfaces gráficas de manejo fácil, la implementación de los Protocolos de Transferencia de Hipertextos (HTTP) como estándar universal para las conexiones de Internet y, junto con ello, el Lenguaje Mark-up de Hipertexto (HTML por

2 Véase la discusión teórica contra el determinismo tecnológico en Schulz (2009). Para un análisis más general del levantamiento zapatista, véase Schulz (1998).

3 La restricción a este periodo se debe a límites de espacio en este artículo. Véase Schulz (2003) para un contexto histórico más amplio, comenzando por la telegrafía en el siglo XIX. 
su sigla en inglés), fueron factores que contribuyeron a un empuje adicional en la propagación del uso de Internet.

Aún así, el acceso a Internet se desplegó de manera dispar. La mayoría de los servidores de Internet y sus usuarios estaban concentrados en las zonas más acomodadas. Hacia finales de 2004, aproximadamente un 85 por ciento de los 265 millones de servidores se encontraban ubicados en los Estados Unidos, Canadá y Europa, mientras que sólo 6.5 millones de ellos se encontraban en América Latina (ITU, 2005). Hoy en día, los países desarrollados tienen en promedio 71.6 usuarios por cada cien habitantes, mientras que en el mundo en desarrollo la cifra es de 21.1 (ITU, 2011).

México fue el primer país latinoamericano que entró a la red. En 1994, de acuerdo a información publicada por la Comisión Federal de Telecomunicaciones mexicana (CFT, 2000) había un estimado de 39 mil usuarios de Internet en México, ${ }^{4}$ lo cual representaba sólo una pequeña fracción de la población mexicana, aunque incluía importantes multiplicadores. El número estimado de usuarios aumentó a 2.9 millones en el año 2000 (Cofetel, 2000), 14 millones en el 2004 (ITU, 2005) y 31.5 millones en el 2009, lo que equivale al 28.3 por ciento de la población (ITU, 2011). Sin embargo, estos datos a nivel nacional no muestran la distribución geográfica dentro del país y en sus diversos estados federales. La información difundida para la reparación de líneas de teléfono celular, no obstante, provee un posible vistazo de las grandes disparidades regionales.

Aún cuando la penetración de las nuevas tecnologías fue aumentando durante el periodo 1994-2004, el promedio para la Ciudad de México siguió siendo cerca de diez veces más alto que el de Chiapas, el estado mexicano con menos conectividad. Incluso, dentro de Chiapas, las líneas telefónicas tendían a estar concentradas en las áreas urbanas mientras que las rurales tenían muy pocas. Las comunidades zapatistas, como la mayoría de los parajes y pueblos de Chiapas, no contaban con líneas telefónicas, en muchos casos ni siquiera tenían electricidad. Las comunicaciones de larga distancia se realizaban por medio de mensajeros o, en algunos casos, en radios bidireccionales de pila.

Los primeros esfuerzos por parte de Organizaciones No Gubernamentales (ONG) mexicanas de utilizar redes informáticas nacionales e internacionales se hallan estrechamente ligados al desarrollo tecnológico de grupos alterna-

4 Otros estimados llegaban hasta una cifra de cien mil (Carson, 1996). Los estimados de cifras de usuarios son notoriamente inexactos. Por lo general, se basan en los recuentos más exactos de los equipos de servidores, éstos se multiplican por un promedio estimado de usuarios que supuestamente comparten un servidor (véase Schulz, 2003). 
tivos en el Silicon Valley en el estado de California, EEUU, y a la resistencia contra el Tratado de Libre Comercio de América del Norte (TLCAN) que provocó la formación de alianzas entre grupos mexicanos, canadienses y estadounidenses. LaNeta fue formada en 1991como ONG pionera de comunicación en línea. En diciembre de 1993 esta organización no gubernamental se afilió a la Asociación para el Progreso de Comunicación (APC) basada en California, obteniendo de ese modo acceso a la red informática nacional e internacional conectada con la APC, incluyendo al Instituto para la Comunicación Global (IGC por sus siglas en inglés), que ya estaba en contacto con diversas ONGs mexicanas desde 1988 (cf. Frederick, 1993; LaNeta, 2000). La ONG Red Mexicana de Acción Frente al Libre Comercio (RMALC) se formó en 1991 a raíz de una reunión de grupos canadienses y mexicanos y conectó a más de cien grupos de movimientos de sindicatos, medio ambiente, mujeres, campesinos y vecinos. La comunicación entre éstos, así como con otros grupos y activistas individuales, fue facilitada enormemente por las nuevas opciones de intercambio vía correo y foros electrónicos (véase Schulz, 2001; Tavera Fenollosa, 2001). Entonces, desde finales de 1993, justo unos meses antes del levantamiento armado de los zapatistas, existían redes electrónicas importantes.

Internet tuvo un papel particularmente significativo en la difusión de información y como vínculo de activistas claves en países más desarrollados y con organizaciones en México. Se utilizó la herramienta del correo electrónico, por ejemplo, para convocar a la "Movilización General Internacional" el 16 de febrero de 1997 al cumplirse el aniversario de los Acuerdos de San Andrés. Hubo movilizaciones simultáneas en numerosas ciudades europeas, australianas y americanas, incluyendo treinta ciudades de los Estados Unidos (Bellinghausen, 1997). El sitio web «http:// www.ezlnaldf.org» fue creado por seguidores zapatistas con el objetivo específico de proveer información actualizada y cobertura detallada de la marcha del EZLN desde Chiapas hasta el zócalo capitalino en 2001. Una consideración más detallada de algunos de los sitios web claves y las listas de correo electrónico nos ayudará a profundizar en cómo funcionaron estas aplicaciones de Internet en tanto herramientas del movimiento zapatista.

\section{Los zapatistas en la Red}

Se configuraron decenas de sitios de Internet para diseminar información acerca del levantamiento zapatista, ${ }^{5}$ la mayoría de los cuales fueron esta-

5 Para un listado amplio de direcciones electrónicas véase Cleaver (1996). 
blecidos y mantenidos por activistas ubicados en países post-industriales. La presentación de la lucha y los objetivos zapatistas dependían de las perspectivas de quien mantenía el sitio web, aunque varios sitios colgaban extensas recopilaciones de comunicados y textos de entrevistas con el Subcomandante Marcos, quien además de políglota también era un portavoz muy capacitado en el manejo de medios de comunicación.

Desde 1994 hasta 2004, uno de los portales zapatistas más complejos se encontraba en la dirección «http://www.ezln.org». Fue configurado en la primavera de 1994 por un joven voluntario, con el consentimiento del EZLN, y en adelante mantenido por Virtual Sites, una filial de ingeniería en internet de la firma GW Communications, instalada en San Francisco (Paulson, 1997). Dicho portal fue originalmente diseñado para poner documentos del EZLN traducidos al inglés a disposición del público angloparlante de los EEUU. A petición de públicos en México y otros países, los textos originales en español fueron posteados, así como sus traducciones a otros idiomas además del inglés. El portal aduce haber recibido más de 725 mil visitas desde octubre de 1996 -fecha en que se equipó el sitio con un contador- hasta el 21 de enero de 2000, lo cual hace un promedio de 18 mil visitas por mes. Las cifras más altas se mostraron durante periodos cercanos a eventos especiales, como la marcha zapatista de 2001 hacia la Ciudad de México. Para julio de 2003, el sitio web aduce haber recibido un total de 4 millones de entradas, cifra que en septiembre de 2005 llegó a más de 5 millones, lo cual ofrece un promedio de más de 40 mil entradas por mes (Paulson, 2005).

Durante la primera etapa de la rebelión, el portal LaNeta ${ }^{6}$ cobró particular importancia. Sus foros electrónicos y boletines de prensa se utilizaron para diseminar rápidamente reportes actualizados a un público interesado en el conflicto de Chiapas. Asimismo, proveía un espacio telecomunicativo para llevar adelante discusiones, posibilitando de ese modo el surgimiento de un grupo activo de seguidores. Luego, LaNeta hizo disponibles importantes recopilaciones de documentos informativos sobre el trasfondo del levantamiento, así como una serie de enlaces o hipervínculos para facilitar el contacto con diversas organizaciones indígenas. La Red de Información para Organizaciones Indígenas, que podía encontrarse a través de LaNeta, comprendía para mayo de 2000, diecinueve organizaciones mayores y menores acerca de las cuales el sitio web ofrecía información detallada (RCI, 2000).

La causa zapatista parecía estar mejor representada en Internet durante los primeros años del conflicto que el mismísimo gobierno mexicano

6 http://www.laneta.apc.org 
gracias al soporte que ofrecían voluntarios capacitados en informática en países post-industriales. Durante los primeros años del levantamiento, los sitios web zapatistas tenían un claro diseño que evidenciaba superioridad tecnológica comparada con sitios oficiales. A las entidades gubernamentales les tomó cerca de un año desarrollar y mejorar sus propios sitios web. El gobierno del estado de Chiapas aduce haber recibido durante el primer año, en su página web, un número cercano a las 37 mil visitas, ${ }^{7}$ mas ello no llegaba a rozar siquiera las cifras de los sitios más importantes del EZLN. El portal del presidente mexicano ${ }^{8}$ estaba mucho mejor diseñado que la página del estado de Chiapas. De hecho, en los años posteriores al gobierno de Ernesto Zedillo (1994-2000), comenzó a ofrecer información más actualizada y detallada acerca de las políticas del gobierno y proyectos, tanto en español como en inglés, así como un archivo en línea de resúmenes de comunicados de prensa, propuestas legislativas, discursos presidenciales, imágenes a color y video clips. También se crearon páginas especiales diseñadas para niños. Había una sección que ofrecía información particularmente abarcadora y completa sobre Chiapas. Los usuarios interesados incluso podían suscribirse a una lista de correo para recibir regularmente los comunicados de prensa más recientes. La presencia gubernamental en Internet se consolidó aún más durante la administración del presidente Fox (2000-2006). ${ }^{9}$ Desde finales de la década de 1990 se fueron creando nuevos sitios web dedicados, por ejemplo, a publicitar los atractivos turísticos del estado de Chiapas, presentando una imagen armoniosa de la región en la que el verdor de la selva y las aguas cristalinas de sus lagos comprendían un deslumbrante primer plano que hacía a un lado cualquier alusión a asuntos conflictivos.

\section{Listas de correo electrónico}

Como método de difusión de una corriente continua de información se utilizaron listas de correo electrónico. Entre éstas destacan Chiapas-L y Chiapas95, ambas basadas en la región fronteriza entre EEUU y México, como las dos más activas y que con frecuencia compartían contenidos. ${ }^{10}$

\footnotetext{
http://www.chiapas.gob.mx

http://www.presidencia.gob.mx

http://www.presidencia.gob.mx y http://www.vicentefox.org.mx

10 Dado que en gran medida la difusión fue automatizada, un número importante de correos electrónicos entraba en circulación consistiendo de sólo un mando como "unsubscribe" o "baja de la lista", o estaban vacíos o no contenían más que una duplicación de una dirección de co-
} 
Chiapas-L fue creada en agosto de 1994 por el colectivo Burn!, un grupo de estudiantes de la Universidad de California en San Diego, con el propósito de ofrecer un foro de discusión sobre eventos relacionados con Chiapas. La lista fue descontinuada cuando la administración de la universidad cerró el servidor debido a quejas respecto a la circulación de comunicados de las Fuerzas Armadas Revolucionarias de Colombia (FARC), los cuales Burn! había en efecto circulado en una lista aparte relacionada con política colombiana. En todo caso, Chiapas-L circuló más de 23 mil correos electrónicos desde su inicio en 1994 hasta finales de 1999, con un volumen anual de comunicación que creció a un ritmo constante incrementando de 3 mil quinientos en 1995 a más de 6 mil cien en 1999. Estas cifras representan un promedio de más de 4 mil quinientos correos electrónicos por año, o cerca de 12.8 por día.

Chiapas95 fue una lista iniciada en el otoño de 1994 por Harry Cleaver, un profesor de economía de la Universidad de Texas en Austin. En febrero de 1995 estuvo completamente habilitada para funcionar. El grupo texano de solidaridad Acción Zapatista se encargó de mantenerla hasta que la labor se volvió geográficamente más dispersa. Aún cuando las discusiones se desarrollaban en la lista los primeros años, con el tiempo se volvió un instrumento más exclusivo utilizado específicamente para la difusión de artículos periodísticos, documentos traducidos, envío de declaraciones y dossiers preparados por ONGs afines a la realidad chiapaneca. Con el paso del tiempo se crearon diversas sub-listas de Chiapas95 para dar a los suscriptores la opción de recibir todos los correos electrónicos, sólo un resumen (Chiapas95.lite), o correos en un idioma específico (Chiapas95english y Chiapas95-español). Más de 47 mil correos electrónicos circularon en la lista principal desde su inicio hasta noviembre de 2005, lo cual representa un promedio de más de 4 mil doscientos correos por año, u 11.7 por día. El volumen de comunicación anual mantuvo un ritmo más bien constante durante los primeros cinco años de operación, con una cifra de 3 mil trescientos correos en 1995 y poco más de 5 mil trescientos en 2000. Este número se duplicó en 2001, el año con el volumen comunicativo más grande, cuando alcanzó los 10 mil. Disminuyó en años posteriores, llegan-

rreo electrónico anterior sin añadir nada nuevo. Para este análisis de los efectos mediáticos, he considerado únicamente aquellos correos electrónicos con un contenido sustancial. Defino "contenido sustancial" de manera amplia para incluir, por ejemplo, correos electrónicos que consisten tan sólo de una frase breve o una palabra despectiva, ya que se puede suponer que en estos casos los correos electrónicos contienen una intención comunicativa y no son meramente producto de un error técnico. 
do a su punto más bajo en 2004 cuando apenas rebasó los setecientos. Este declive podría ser indicativo de un agotamiento luego de la alta de 2001, no obstante, la cifra se eleva nuevamente en 2005 cuando llega a los mil quinientos. El alto nivel de volumen de comunicación anual y su reciente recuperación sugieren que el interés en el tema no era cuestión de moda pasajera. El volumen de comunicación no se disipó después del auge inicial de interés, sino que mantuvo un ritmo sostenido por más de una década.

En ambas listas la mayoría de los correos electrónicos eran en inglés o español, aunque ocasionalmente aparecían correos en otros idiomas tales como el alemán. Con base en una muestra aleatoria $(\mathrm{N}=119)$ tomada de correos electrónicos en el verano de 1999, se puede estimar que cerca de la mitad de todos los correos electrónicos distribuidos y clasificables fueron enviados desde direcciones canadienses o estadounidenses. Por su parte, un tercio de todos los e-mails distribuidos y clasificables fueron enviados desde servidores mexicanos. El resto se originó en otros países latinoamericanos (4 por ciento) y europeos (11 por ciento) con un alto porcentaje proveniente de España ( 5 por ciento).

Aproximadamente, dos tercios de los correos electrónicos que fueron recolectados de ambas listas hasta septiembre de 1999 eran reportajes de agencias noticiosas $\mathrm{u}$ otros medios, tales como el periódico mexicano $\mathrm{La}$ Jornada. Alrededor de la mitad del tercio restante de los correos electrónicos concernían a otras actividades, incluyendo anuncios de eventos especiales, llamados para demostraciones y cartas de protesta e información sobre campañas. Una sexta parte de los correos electrónicos consistía en análisis, evaluaciones y contribuciones al debate.

Es difícil estimar el número de lectores de los correos electrónicos debido a que eran usualmente reenviados a un sinnúmero de listas formales e informales. El número y los nombres de los suscriptores registrados en Chiapas-L y Chiapas95 fluctuaban considerablemente. En el verano de 1999, Chiapas-L tenía por lo menos 177 suscriptores registrados (día de conteo: 3 de julio de 1999) y Chiapas95, 312 (día de conteo: 5 de julio de 1999). Considerando el gran volumen de comunicaciones, podría deducirse que los suscriptores a largo plazo de Chiapas-L y Chiapas95 debieron estar significativamente interesados en Chiapas como para haber mantenido el contacto a través de semejante cantidad de mensajes. Cabría suponer que algunos de los activistas más comprometidos así como multiplicadores pertenecían a este grupo.

En la medida en que las direcciones de correo electrónico de suscriptores registrados el día del conteo puede servir como indicador de su ubicación geográfica, se observa que la gran mayoría (el 84 por ciento) provenía 
de países del mundo post-industrial (Estados Unidos, Canadá, Europa, Australia, Japón) donde el dominio educacional americano (sufijo ".edu") se presenta en cerca de dos tercios de todas las direcciones localizables. Solamente el 20 por ciento de las direcciones de correo electrónico localizables pertenecían a México (sufijo ".mx"), mientras que el otro 2 por ciento pertenecía a otros países de América Latina. No aparecía representación de los países en desarrollo no latinoamericanos.

Pese a la base estable del volumen de comunicación anual en Chiapas95 y Chiapas-L, la frecuencia de comunicación tanto diaria como mensual muestra altas y bajas significativas. Tales variaciones dan lugar a cuestionarse sobre la intensidad de la comunicación, ¿de qué depende?, ¿de los eventos de Chiapas propiamente, o de los tiempos y calendarizaciones de los activistas internacionales? El análisis comparativo de la curva de frecuencia de comunicación y la cronología de los sucesos en el contexto del levantamiento podría ofrecer una posible respuesta.

Dicha comparación muestra que la frecuencia de comunicación aumentaba cuando el conflicto se intensificaba, o cuando se llevaban a cabo eventos especiales. La frecuencia de comunicación fue particularmente intensa en febrero de 1995. Correspondió con la segunda ofensiva del gobierno mexicano, la cual fue casi inmediatamente seguida de manifestaciones de protesta internacional antes de su colapso. Otro pico de comunicación tuvo lugar en agosto de 1996. Esta fue la fecha en que se realizó el Encuentro intercontinental contra el neoliberalismo y por la humanidad en Chiapas. Un pico aún más pronunciado ocurrió en diciembre de 1997 en virtud de la masacre de Acteal, en la que cuarenta y cinco campesinos de una pequeña comunidad desafiaron al PRI local y fueron asesinados, este suceso desató una ola de acciones solidarias. La curva de frecuencia de comunicación para Chiapas95 presenta una interrupción durante septiembre de 1998. No se enviaron correos electrónicos desde Chiapas 95 en tal fecha pues la lista se encontraba en estado de disolución temporal dado que los activistas que la habían instalado y operado hasta ese entonces carecían del tiempo necesario para continuar con la tarea. A unas pocas semanas, sin embargo, apareció un nuevo grupo de voluntarios que retomó las riendas de ésta. Seis años después, las actividades de la lista se vieron interrumpidas nuevamente, pero en esa ocasión pasaron cinco meses antes de que las actividades retomaran su ritmo regular. No obstante, el punto más intenso de actividad corresponde al 2001, año que coincidió con la marcha zapatista hacia la Ciudad de México. La marcha generó gran volumen de comunicación durante las semanas y meses que siguieron al anuncio inicial del evento, así como durante los sucesos mismos de la campaña la cual culminó con dis- 
cursos de dirigentes del EZLN frente de una multitud de 200 mil personas en el zócalo capitalino y luego en el pleno del Congreso de la Unión. En fin, estos ejemplos ilustran cómo la frecuencia de comunicación puede ser explicada como resultado de los eventos que acontecían en Chiapas en conjunción con las dinámicas de grupos de activistas internacionales. El flujo estable y continuo de información servía para mantener amplias y diversas redes de vínculos a través del tiempo y espacio que podían ser activados para la rápida diseminación de información y / o búsqueda de mayor apoyo en momentos de gran intensidad de actividades in situ del movimiento.

Un vistazo a los ejes temáticos de las comunicaciones en Chiapas-L y otras listas de discusión, así como de sub-listas relacionadas con el conflicto de Chiapas, muestra cómo algunas de las características estructurales conocidas por los medios masivos de comunicación se dan también en Internet. Por ejemplo, se observan relaciones de comunicación asimétricas, así como la falta de disposición para considerar ciertos argumentos y perspectivas de informaciones equívocas, entre otros, como obstáculos a la comunicación. Las tecnologías de comunicación interactiva proveen a los actores sociales de una gama diversa de canales y espacios de expresión. Sin embargo, no garantizan que el discurso que surge de ello corresponda con una situación discursiva ideal, libre de cualquier instancia de relación de dominación. La antropóloga Hellman (2000), por cierto, ya había advertido que el discurso del activismo internacional respecto al conflicto en Chiapas con frecuencia era superficial, unilateral y se mostraba a la merced de rumores sin fundamento (véase también Cleaver, 2001; Paulson, 2001; y Hellman, 2001). Por supuesto, la calidad de la información que circula corresponde a la calidad de sus fuentes y también refleja la particular perspectiva de éstas. No obstante, y pese a obstáculos relacionados con precisión y asimetrías de poder, la dinámica de las listas de correo electrónico y las conversaciones que generaron ciertamente sirvieron para difundir el punto de vista de los zapatistas de manera efectiva y eficiente a un público transnacional.

\section{Conclusiones}

En esta sección sintetizaré los hallazgos derivados del estudio de caso en términos de diez puntos analíticos. Luego concluiré con una breve consideración de sus implicaciones estratégicas a fin de plantear ciertas observaciones meta-teóricas que podrían orientar la investigación a futuro. 
1. Internet contribuyó a socavar los esfuerzos por parte del gobierno de moldear la opinión pública al controlar de maneras diversas el material publicado en los medios de comunicación, aunque no de manera tan abarcadora como se ha propuesto comúnmente. Discusión: Sí, Internet sin duda fue instrumental en la difusión del mensaje de los insurgentes, logrando socavar el fuerte control gubernamental sobre los medios de comunicación. De ese modo alimentó el intercambio de opiniones controvertidas, dando lugar a cierto pluralismo de perspectivas. Por consiguiente, el gobierno mexicano no tuvo éxito al imponer su representación original del levantamiento. Pero ello no puede ser atribuido solamente al impacto de Internet, sino que debe ser visto en su propio contexto en términos del proceso general de democratización y liberalización de los medios mexicanos. Diarios tales como La Jornada y Reforma, así como el semanario Proceso, ya habían logrado un importante margen de independencia de la perspectiva oficial y gozaban de un amplio público lector instruido, aunque no tan abarcador como los de la radio y televisión, medios de comunicación masiva estrictamente más controlados. El diario La Jornada, en particular, parece haberse beneficiado de la cobertura de la insurgencia zapatista ya que la venta de ejemplares creció significativamente, lo que le permitió solventar la crisis financiera que enfrentaba en la época. El control de los medios electrónicos de comunicación en México era muy fuerte a comienzos de la insurgencia en 1994. Esto cambió con la derrota electoral del régimen del PRI, como lo ilustra, por ejemplo, la entrevista al Subcomandante Marcos realizada por el fundador de la revista Proceso, Scherer García, la cual fue transmitida por Televisa, canal televisivo que anteriormente estrechaba fuertes lazos con las posiciones del gobierno de turno.

2. El impacto de Internet en México ha sido limitado por su bajo índice de penetración.

Discusión: Aún en 2010 se estimaba que entre un cuarto y una tercera parte de la población mexicana era usuaria de Internet. Para la época del levantamiento zapatista en 1994, se estimaba que en una población de 100 millones de personas sólo 39 mil individuos tenían acceso básico a la red informática. No hay datos disponibles que muestren exactamente los patrones de distribución de las cifras de usuarios en el país, sin embargo, a falta de ello, los datos sobre la posesión de equipo y la disponibilidad de líneas te- 
lefónicas podrían ser referentes útiles pues proveen información que posiblemente apunte a ciertos patrones de distribución. Éstos indican que el acceso a Internet se ha concentrado y continúa concentrándose entre los sectores poblacionales de mayor nivel educativo y económicamente pudientes en las ciudades, mientras que es prácticamente inexistente entre los pobres rurales, a excepción de casos en los que se observa cierta concentración en los que el acceso podría haber sido facilitado por ONGs, proyectos piloto del gobierno o de turismo.

3. ElpapeldeInternetera menosimportantedentro deMéxicoqueanivel global.

Discusión: Dentro de México, el impacto de Internet era limitado debido al bajo índice de penetración y poca importancia en comparación a los medios impresos tales como La Jornada, dedicados a cubrir los eventos desde una perspectiva independiente del régimen. Sin embargo, Internet desempeñó un papel importante en la labor de atraer públicos más allá de las fronteras de México, especialmente en otros países de la Organización de Cooperación y Desarrollo Económico (OCDE). Aunque los medios de comunicación ocasionalmente ofrecían reportajes noticiosos sobre la insurgencia zapatista en dichos países, los sitios web y listas de correo electrónico en Internet proporcionaban información mucho más amplia; en especial desde la perspectiva de los insurgentes, casi a diario tanto en español como en inglés y en ocasiones hasta en alemán e italiano, entre otros. Las iniciativas zapatistas sirvieron como catalizadores e impulsaron una comunicación transnacional que incluso fue dando lugar a la formulación de debates respecto a las dinámicas y los procesos de globalización entre actores de una incipiente sociedad civil global. A su vez, las nuevas redes transnacionales contribuyeron a las manifestaciones y marchas que se realizaron durante cumbres de la élite global en Praga, Seattle y Génova. Al mismo tiempo, laboraban para mantener la atención global de manera sostenida sobre el conflicto en Chiapas a fin de ejercer una presión e influencia que lograra limitar las opciones militares del gobierno mexicano como herramienta para lidiar con la insurgencia zapatista (Schulz, 2001b). 
4. Pese a que Internet no alcanzó una audiencia de masas, desempeñó un papel instrumental como vía para conectar a activistas y multiplicadores clave

Discusión: La baja tasa de penetración de Internet apunta al hecho de que no había un público de masas para los mensajes distribuidos en la red o a través de las listas de servidores. No obstante, como muestran las entrevistas a activistas clave internacionales, los principales medios para obtener información sobre eventos locales (en México) en tiempo real, fueron interfaces de Internet. Dichos activistas se mantuvieron al tanto de los acontecimientos por medio de los miles de mensajes que recibían al mes. A su vez, difundían en sus redes locales información sobre aquellos eventos que juzgaban más significativos a través del reenvío de información selecta o también por medio de reuniones en persona. Aquellos que mantenían los sitios web y las listas de servidores también tenían control sobre cómo se perfilaba la imagen del movimiento. Discusión: Los voluntarios que poseían las destrezas requeridas, el tiempo y los recursos para instalar y mantener sitios web y listas de e-mails, tenían la habilidad de crear o seleccionar el material que enviaban por correo o posteaban. El servidor de la lista y los administradores de los portales posteaban frecuentemente todo el material que los activistas-seguidores enviaban sin editarlo. Una gran cantidad del material que circulaba por los servidores de listas consistía en artículos traducidos de La Jornada, el periódico mexicano suprarregional de izquierda con la cobertura más abarcadora del conflicto en Chiapas. Los sitios web y servidores de las listas mantenidos por grupos de activistas de una universidad californiana fueron clausurados cuando un empleado se sintió amenazado por un reclamo oficial de Colombia sobre una cobertura que simpatizaba con el conflicto en dicho país.

5. Los actores de base llevaban la delantera en Internet pero con el paso del tiempo el gobierno mexicano actualizó sus modalidades de comunicación y se puso al corriente con el mundo de la informática. Discusión: Los voluntarios-activistas ubicados en ciudades norteamericanas y europeas utilizaron listas de servidores de correo y sitios web previamente establecidos y también lograron formular nuevos listados y sitios al poco tiempo de suscitarse el levantamiento zapatista. Al gobierno mexicano le tomó cerca de un año crear una propaganda propia en sus sitios web. Sin embargo, gracias a 
los vastos recursos a su disposición, logró desarrollar su presencia informática de manera acelerada y efectiva en un espacio de poco tiempo. Hoy en día, la mayoría de los sitios web relacionados con Chiapas presentan un claro vínculo comercial y en su mayoría se encuentran dedicados a promover el turismo en la región.

6. Las características interactivas de Internet no fueron utilizadas al máximo de sus posibilidades.

Discusión: El análisis de contenidos de muestras representativas de los más de 40 mil correos electrónicos distribuidos por las principales listas de correo, indica que los potenciales interactivos que ofrece Internet no se utilizaron tan eficiente y efectivamente como podrían haberlo sido. La mera existencia de infraestructuras interactivas no garantiza una situación ideal de habla (véase Habermas, 1981).

7. A nivel local, la radio fue mucho más importante que Internet.

Discusión: A pesar de que la gran mayoría de los sitios vinculados a la insurgencia estaban y siguen estando basados fuera de México, algunos de los sitios estaban ubicados en Chiapas. Sin embargo, debido a la poca accesibilidad a Internet en las zonas rurales de Chiapas, la radio de base comunitaria resulta el medio más importante a nivel local.

8. La comunicación digital facilita nuevas formas de vigilancia.

Discusión: Entre activistas mexicanos se ha expresado la preocupación de que las comunicaciones pudieran estar siendo monitoreadas. Aún cuando no hay evidencia específica y comprobada al respecto, la experiencia en otros países sugiere que dicha preocupación no carece totalmente de fundamento. Tanto Internet como la telefonía móvil son medios que dejan huellas de sus comunicaciones. El gobierno chino, por ejemplo, ha utilizado información de usuarios de Internet para arrestar disidentes. Por otra parte, en EEUU, tuvo lugar un caso en que se comprobó que administradores escolares vigilaban las actividades de alumnos en sus casas a través de las cámaras web con las que estaban equipadas las computadoras portátiles que la escuela facilitaba a los estudiantes. Más recientemente, en el arresto de un docente universitario en Alemania salió a relucir que se convirtió en sospechoso debido a que se comunicaba a través de cuentas anónimas de correo elec- 
trónico en cibercafés y además, en los textos de sus comunicados utilizaba términos críticos menos conocidos como "gentrificación".

9. El impacto más importante de Internet en México no fue la reducción sino la agudización de la desigualdad social.

Discusión: Si bien Internet sirvió como herramienta efectiva para la difusión de la causa y el mensaje zapatista al lograr atraer un grupo considerable de seguidores y simpatizantes más allá de las fronteras mexicanas, el efecto más palpable de la introducción de Internet en el país, fue el surgimiento de nuevas desigualdades sociales pues el acceso a la red informática se desarrolló a partir de mecanismos de mercado y no según pautas de objetivos sociales. Por consiguiente, sólo aquellos que podían costear las nuevas tecnologías gozaban de acceso a la red global de información y comunicaciones; los que no, quedaban rezagados y paulatinamente marginados. Como resultado, las desigualdades sociales existentes se agudizaron. ${ }^{11}$

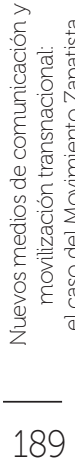

Estos hallazgos han de constituir un llamado a la cautela en el momento de sobre o subestimar el papel liberador que se ha adjudicado a los nuevos medios. La eficacia de una interfaz mediática específica depende del tiempo y del contexto. Los públicos locales, nacionales, regionales y transnacionales interactúan a través de diferentes interfaces mediáticas. Sólo una pequeña parte de la población mexicana tenía acceso y contaba con servicio de Internet al inicio del levantamiento zapatista, y aún hoy en día, las cifras de difusión siguen siendo bajas en comparación con otros países de la OCDE. Internet facilitó las comunicaciones a través de fronteras nacionales y grandes distancias, ayudó a establecer y mantener vínculos estrechos que se movilizaban eficaz y rápidamente en ciertos momentos de necesidad particular y fue herramienta útil en tanto red para la coordinación de actividades transnacionales. Los activistas del norte global ${ }^{12}$ tenían mayor capacidad de acceso a equipo informático (computadoras, etc.), a conexiones más veloces y, además, las destrezas tecnológicas de punta necesarias para perfilar la imagen del movimiento en el nuevo medio, para bien o para mal. La calidad participativa de los debates y discusiones en el nuevo medio con frecuencia no utilizaron las características interactivas que ofrecía Internet al máximo de sus posibilidades. Aun así, el flujo regu-

11 Véase Schulz (2003) para una discusión más amplia sobre este punto.

12 En este caso principalmente activistas de Estados Unidos, Canadá y Europa occidental. 
lar y continuo de información contribuyó a mantener vínculos estrechos que fueron movilizados en momentos en que el conflicto en Chiapas se intensificaba o en los que se llevaban a cabo eventos especiales. Durante el primer año y medio del levantamiento zapatista, el gobierno mexicano se encontraba en desventaja respecto a los activistas en el ciberespacio; sin embargo, debido a la riqueza de recursos a su disposición, en poco tiempo logró desarrollar su presencia informática de manera acelerada y efectiva para posicionarse a la par de dichos activistas. La radio comunitaria, ahora también parcialmente disponible a un público más amplio a través de Internet, fue particularmente útil para los grupos marginados de fuerte tradición oral dado que fomentaba tanto la comunicación interna como la divulgación de información a actores en otras localidades. Dentro de estas consideraciones, es importante tener en cuenta que si bien Internet puede resultar una herramienta significativa para los movimientos sociales, también ha dado lugar a la desigualdad digital y ha suscitado riesgos de nuevos controles. Este estudio de caso resalta tanto las nuevas oportunidades como los límites y riegos de los nuevos medios para el activismo y los movimientos sociales.

En términos generales, el estudio de la comunicación política en el contexto del levantamiento zapatista demuestra que Internet generó, en conjunto con otros medios, tanto poder comunicativo a nivel transnacional que el gobierno se vio obligado a tomarlo seriamente en cuenta. La globalización de los canales de comunicación hizo imposible que un solo régimen pudiera desconectarse del intercambio de ideas a nivel mundial. La difusión acelerada de Internet y otras tecnologías asociadas obstaculiza cada día más los esfuerzos por parte de los Estados-nación por controlar la expresión y diseminación de la opinión pública. Considerando la competencia por la atención pública que se ha originado con la creciente comercialización dentro los nuevos medios, hay que tener en cuenta el costo creciente de recursos destinados al diseño profesional de éstos. El fenómeno de la mezcla de prensa amarillista y de alta calidad que se observa en los medios impresos también aparece en las dinámicas de información disponible en Internet multimedia. La sociedad civil global basada en el reconocimiento universal no fluye automáticamente a partir de la construcción de canales de telecomunicación interactiva. La difusión de una conciencia cosmopolita no sólo depende de la disponibilidad y acceso a canales de comunicación, también, y de modo significativo, de la manera en que se utilizan dichos canales.

¿Puede el uso de Internet por el movimiento zapatista servir como modelo para otros grupos en América Latina o en el mundo? Por una parte 
sí y, por otra, no. El éxito del movimiento zapatista dependió en gran parte de una confluencia históricamente única de diversos factores políticos específicos que han cambiado en la actualidad (véase Schulz, 1998). Los activistas zapatistas informáticos tenían en su favor la novedad. El gobierno mexicano se mostró inicialmente lento en su respuesta propagandística, pero con el tiempo fue capaz de movilizar muchos más recursos en la lucha por acaparar la atención del público. De hecho, hay que ver cómo actualmente en los resultados de los motores de búsqueda, la información comercial acerca de Chiapas curiosamente opaca las páginas de activistas. Con el tiempo toda innovación tiende a perder el elemento sorpresa del que deriva su vigor inicial. La lección general que ofrece el caso zapatista es que el poder de los de abajo proviene de su imaginación creativa. Si bien las tácticas específicas de los zapatistas pueden haber perdido poder de impacto al ser emuladas, en lo que se refiere a la creatividad como elemento estratégico, el caso del movimiento zapatista puede servir como modelo.

El hecho de que el gobierno mexicano se diera cuenta que apremiaba la necesidad de mejorar sus estrategias de relaciones públicas no constituyó un elemento sorpresa, dado que sólo era cuestión de tiempo en lo que surgía algún actor de mayores recursos posicionado como competencia en tecnología y diseño. Lo mismo vale para otros actores políticos, sociales o económicos. Estas observaciones deberían incluso ser consideradas como una llamada a la cautela para no sobreestimar la capacidad de Internet como herramienta para atraer público a campañas de movilización social. A medida que crece la red informática de Internet, la tarea de discernir la información útil de la no deseada se torna cada día más difícil. Los portales y motores de búsqueda colaboran en el proceso de selección de acuerdo a una lógica programada. Por otra parte, aquellos que buscan direcciones electrónicas específicas pueden lograr el acceso la mayoría de las veces, en forma instantánea.

El caso zapatista muestra cómo los públicos locales, nacionales, regionales y globales se hallan interrelacionados y cómo las diversas interfaces mediáticas operan simultáneamente. El impacto de Internet no debe ser exagerado ni subestimado. El flujo constante de información que circulaba en listas de e-mails y sitios web sirvió para crear el público disidente transnacional así como los vínculos latentes que se movilizaron en momentos clave. La mera existencia de interfaces mediáticas interactivas no garantiza de por sí el surgimiento de una sociedad civil global basada en el reconocimiento mutuo. La tolerancia, el respeto y la solidaridad no sólo dependen del formato de los canales de comunicación, también de la manera en que éstos se utilizan. 


\section{Bibliografía}

Albrow, Martin, 1997, The Global Age: State and Society Beyond Modernity, Stanford, California: Stanford University Press.

Carson, Teresa, 1996, "Internet's Popularity Leaping in Latin America”, Miami: Reuters. 6 de junio.

Castells, Manuel, 2002, La era de la información. Vol. II: El poder de la identidad, México, Distrito Federal: Siglo XXI Editores. [Original inglés 1997.]

Castells, Manuel, 2001, La era de la información. Vol. I: La sociedad red, México, Distrito Federal: Siglo XXI Editores. [Original inglés 1996.]

CFT (Comisión Federal de Telecomunicaciones), 2000, Informe de labores 2000, México, Distrito Federal: CFT.

Christian Science Monitor, 1995, "Marcos on the Internet" [editorial], 27 de febrero 1995, p. 20.

Cleaver, Harry, 2001. “The Virtual and Real Chiapas Support Network: A Review and Critique of Judith Adler Hellman's 'Real and Virtual Chiapas: Magic Realism and the Left', Socialist Register, 2000. http://www.eco.utexas.edu/facstaff/Cleaver/anti-he (Consultado el 23 de septiembre de 2005).

Cleaver, Harry, 1996, "Zapatistas in Cyberspace: A Guide to Analysis \& Resources" http://www.eco.utexas.edu/faculty/Cleaver/zapsincyber.html (Consultado el 13 de noviembre de 1997).

Cleaver, Harry, 1995, “The Zapatistas and the Electronic Fabric of Struggle” http:/ / www.eco.utexas.edu/faculty/Cleaver/zaps.html (Consultado el 13 de noviembre de 1997).

Domínguez, Ricardo, 2002, “Digital Zapatismo." http://www.thing.net/ rdom/ ecd/DigZap.html (Consultado el 9 de Septiembre de 2002).

Fox Quesada, Vicente, 2000, “Latin America's Technology Revolution” (Vicente Fox Quesada, interviewed by Alison Bay, 1998), Government Technology http: // www.govtech.net/publications/visions/augvision/fox/fox.phtr (Consultado el 12 de diciembre 2000). 
Frederick, Howard, 1993, "Computer Networks and the Emergence of Global Civil Society" En: Global Networks: Computers and International Communication, Linda M. Harasim (ed.), Cambridge, Massachusetts, MIT Press.

Habermas, Jürgen, 1981, Theorie des kommunikativen Handelns, 2 vols. Fráncfort del Meno, Alemania, Suhrkamp.

Hellman, Judith Adler, 2001, “A Reply to Paulson”, Socialist Register.

Hellman, Judith Adler, 2000, "Real and Virtual Chiapas: Magic Realism and the Left," Socialist Register.

ISC (Internet Systems Consortium), 2011, "ISC Internet Domain Name Survey." http:/ / www.isc.org (Consultado el 15 de junio de 2011).

Ishay, Micheline R., 1995, Internationalism and Its Betrayal, Minneapolis, University of Minnesota Press.

ITU (International Telecommunications Union), 2011, World Telecommunication Indicators Database, Ginebra, Suiza, ITU.

ITU (International Telecommunications Union),2005, World Telecommunication Indicators Database, Ginebra, Suiza,ITU.

Keck, Margaret y Kathryn Sikkink, 1998, Activists Beyond Borders: Networks in International Politics, Ithaca,Cornell University Press.

Lane, Jill, 2003, “Digital Zapatistas”, The Drama Review, vol. 47, núm. 2, pp. 129-144.

LaNeta, 2000, "Programa LaNeta", http: //www.apc.org/home.htm (Consultado el 22 de mayo de 2000).

McLuhan, Marshall, 1964, Understanding Media: The Extensions of Man, New York, Mentor.

Paulson, Justin, 2001, "Peasant Struggles and International Solidarity: The Case of Chiapas", Socialist Register.

Paulson, Justin, 1997, “About ‘Ya Basta!, http: //www.ezln.org” (Consultado el 19 de noviembre de 1997)), http: / / www.ezln.org/about.html (Consultado el 12 de diciembre de 1997). 
RCI (Red de Información para Organizaciones Indígenas), 2000, “Organizaciones integrantes de la red", http: //www.laneta.apc.org/rci/organinteg/organ. html (Consultado el 22 de mayo de 2000).

Robberson, Tod, 20 de febrero de 1995, “Mexican Rebels Using A High-Tech Weapon",The Washington Post, , pp. A1, A21.

Ronfeldt, David y John Arquilla, 1998, The Zapatista Social Netwar in Mexico. Santa Mónica, CaliforniaRAND.

Rovira Sancho, Guiomar, 2009, Zapatistas sin fronteras: Las redes de solidaridad con Chiapas y el altermundismo, México.Era.

Schulz, Markus S., 2011a, "Values and the Conditions of Global Communication" Current Sociology, vol. 59, núm. 2, pp. 238-251.

Schulz, Markus S., 2011b, “The Values of Global Futures”, Current Sociology, vol. 59, núm. 2, pp. 268-272.

Schulz, Markus S., 2009, "Structured Modes of Interplay and the Modeling of Digital Futures", en: The ISA Handbook of Contemporary Sociology, Devorah KhalekinFishman y Ann Denis (eds.), Londres, Sage, pp. 291-304.

Schulz, Markus S., 2003, Internet und Politik in Lateinamerika, Vol. IV: Mexico zwischen Liberalisierung und Demokratisierung, Francfort de Meno, Alemania, Vervuert.

Schulz, Markus S., 2001a, "Democracia y cambio de los medios en América Latina", Perfiles Latinoamericanos, vol. 10, núm. 18, pp. 9-28.

Schulz, Markus S., 2001b, "Political Violence, Human Rights, and Military Strategy," Forum International, Berlín, Alemania, SOWI, vol. 22, pp. 293-328.

Schulz, Markus S., 1998, "Collective Action Across Borders: Opportunity Structures, Network Capacities, and Communicative Praxis in the Age of Advanced Globalization", Sociological Perspectives, vol. 41, núm. 3, pp. 587-616.

Tavera Fenollosa, Ligia, 2001, “Creando redes electrónicas desde y para la sociedad civil", Perfiles Latinoamericanos, vol. 9, núm. 18. 